\section{Hans Åhlfeldt, ove Wigertz}

Dept of Medical Informatics, Linköping University, Linköping, Sweden

\title{
Education and Training
}

\section{Study Programs in Medical Informatics at Linköping University}

implementation of medical informatics curricula. A group at the level of the National government has focused on information technology (IT) and its social and economic impact on society. The school system and the area of health care, among others have been identified as key sectors for the utilization of modern IT. IT is seen as the backbone technology for promoting information retrieval and information exchange between individuals and organizations, and as a means of enhancing the capability of acquiring knowledge from various sources, independently of constraints with respect to location and time. The expectation is that the use of IT will increase the quality, improve the efficiency and reduce the cost-benefit ratio of health care services. Another group, consisting of teachers and researchers within the medical informatics area, who represent all the major universities in Sweden, have surveyed the status of medical informatics within the medical faculties in Sweden. The group have stressed the increased need for health care professionals at different levels to acquire competence and skills with respect to IT, and they have identified the following areas as main topics for medical informatics education: * basic computer literacy including common software such as wordprocessing, spreadsheets, graphics, etc.,
* information retrieval and communication through networks, especially utilization of tools for accessing library services and public networks such as Internet,

* computer-based medical record systems including database structure, and demands on terminology and coding schemes,

* quality assurance of medical procedures including methods for database querying, basic statistics and computer-based medical decision support.

\section{Medical informatics and biomedical engineering as Master of Science subjects}

The Master of Science (M.Sc.) education programs in biomedical engineering and medical-informatics are closely related. Basic parts of both programs are devoted to areas of medicine such as cell biology, anatomy and physiology, and to subjects such as ethics, health care organization and clinical medicine. The latter comprises methods used in diagnostics and therapeutics and aims at an understanding of problems in patient management. Co-operation among clinical disciplines is also explained and demonstrated here. Specialization in biomedical engineering or medical informatics depends on the courses chosen in the 
department as well as on whether courses in computer science or engineering mechanics and electronics are preferred. Composition of the M.Sc. program is shown in figure 1 . The education is mainly aimed at preparing graduates to be able to work in health and medical care as clinical or system engineers in hospitals and in industrial health services. Research and teaching activities and the manufacturing of medical equipment are also important markets for graduate engineers.

The list of courses within the field:

* Biomedical Engineering, which provides a broad introduction to the field.
* Anatomy and Physiology, which provides knowledge about the organs in the human body, can include medical terminology.

* Physiological Pressures and Flows, which provides models and measurement techniques for the circulatory-, respiratory-, and digestive organs.

* Biomedical Transducers, which provides practical experience in the development of transducers and measurement systems for different physiological parameters.

* Intensive Care and Rehabilitation, which focuses on methods and systems for monitoring critically ill patients, models and techniques for rehabilitation, and assessment of

\section{Master of science in medical informatics and biomedical engineering}

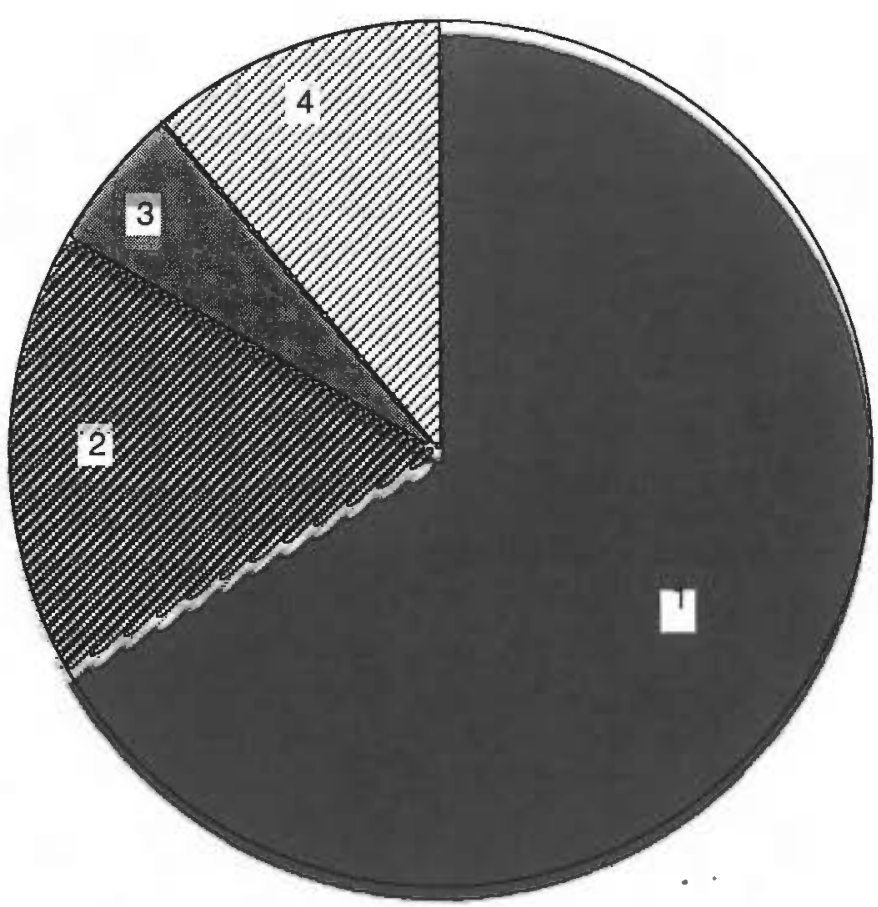

Figure 1. Composition of the Master of Science program ( 4.5 years) in medical informatics and biomedical engineering at Linköping University. 1) Three years (120 credits) with training in mathematics, physics, electronics, and computer science, 2) courses within the fields of medical informatics and biomedical engineering, 3) elective courses, 4) Master's Thesis. One credit corresponds to one week of full-time studies. medical technology.

* Technical Systems in Health Cary which covers safety regulation norms, and standards in relation to medical technology.

* Biomedical Signal Processing which covers the genesis and infor mation content of biomedical nals with application of a variety signal processing methods.

* Medical Imaging, which covers all image modalities used within medj. cine including PACS (pictury archiving and communication sys. tem).

* Medical Informatics, which covers computer-based medical recordy and architecture of modern hospital information systems.

* Computer Assisted Decision Mak. ing in Medicine, which focuses on models for medical decision making and surveys of methods for med. cal decision support.

In additions to lectures and nars, all courses contain hands-on ${ }_{\text {train }}$ ing with different computer applicas tions, shorter laborative components or longer projects. To a large exten, the curricula for courses within medical informatics are project-orienter The lectures aim at giving a survey of the field, whereas the projects provid deeper insight into some of the blocks in figure 2. The projects are form lated in part by the students the selves and their tutor, and the grof size is about 3-4 students.

\section{Medical informatics for medical students.}

Before 1984 medical informatic was taught to medical students Linköping University during the " $\mathrm{cl}$ " cal" period and mostly through sing lectures that were squeezed into th tight traditional curriculum.

After that date the Medical Faculte 


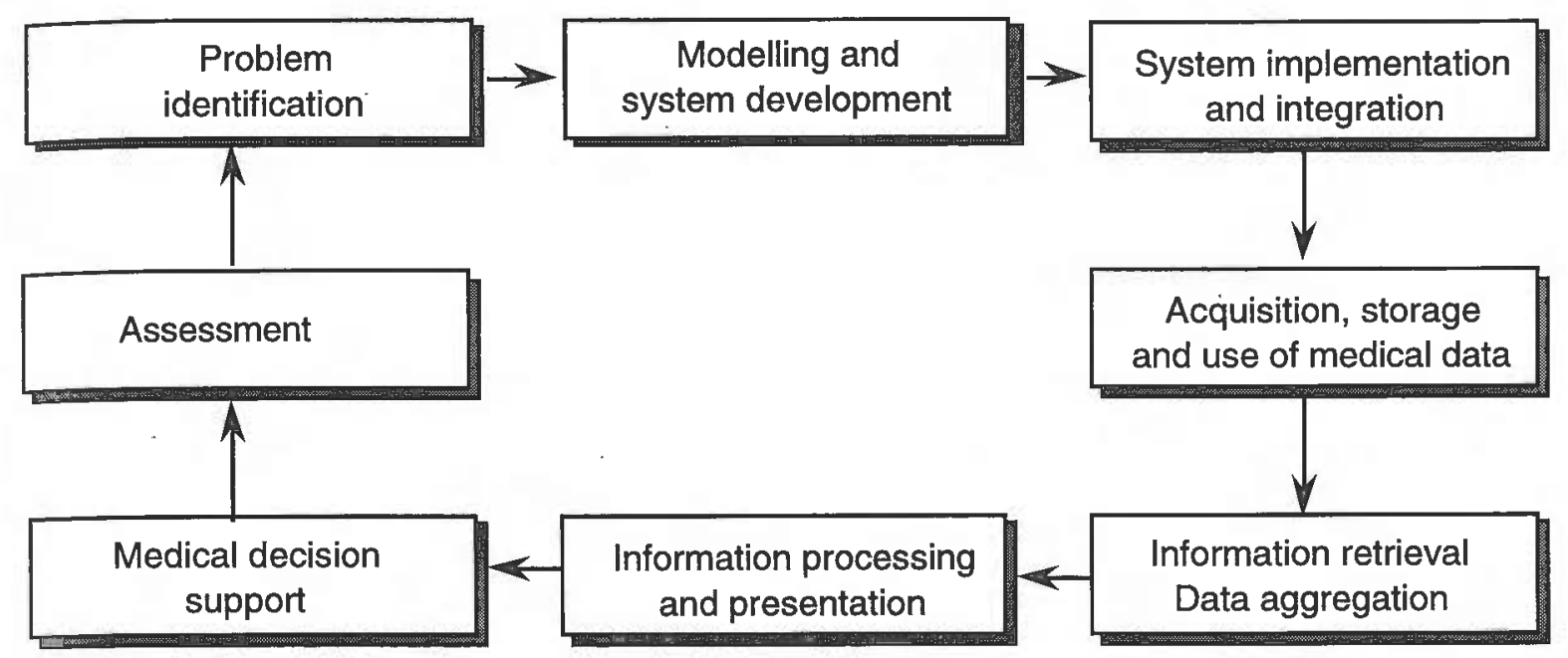

Figure 2. Process-oriented view of the subject medical informatics with the overall objective of improving information management in order to increase quality and decrease the cost-benefit ratio of medical procedures (interventions, therapy, services, etc.).

became Linköping Faculty of Health Sciences with a united organization between the university and the county council, providing the education of laboratory technicians, nurses, occupational therapists, physicians, physiotherapists and supervisors for social service and care. Integration between courses, training in team work, contact with research, problem-based studies, early contact with patients and emphasis on prevention and health promotion are characteristics of the educational philosophy.

The Department of Medical Informatics was then also engaged in redesigning the education in medical informatics so it would conform better with and be integrated into the changed form and concept of the new medical and health care education that was introduced.

The program for medical studies is Prganized to give the student competence in making decisions and solving medical problems. To fulfil this, the student must acquire an amount of nowledge, skills and attitudes. The cientific basis for medical decisions is undergoing rapid development and change. Therefore, an important principle of the program is to develop the ability for lifelong, independent learning and to understand the principles of medical decision making which are taught in medical informatics. Such competence means that the student should be able to

- Identify his/her learning needs

- Choose and use various sources of information

- Evaluate critically sources of information

- Evaluate his/her learning in relation to the needs

The educational methods used have been chosen to train the student in the skills listed above. The learning is directed by objectives. The student bears the main responsibility for reaching the competence set and has much freedom in choosing between various learning resources. Teachers and tutors will stimulate and guide such an active learning in various ways.

The studies are problem based to a large extent. Computer based cases and real patients are used to focus on problems in human biology, clinical medicine, community medicine and behavioural science in a context which the student will meet in his/her future work [4]. The students work on these problems in small groups with a tutor.

Staff from theoretical and clinical disciplines will share the tutorship during the whole program. This way of problem based learning is similar to research and clinical problem solving. This new concept of medical and health care education is strongly connected to the progress in the fields of medical informatics, computer communication and information retrieval. Several important functions have been identified in an effort to create distributed tools for information and knowledge retrieval, such as easy-to-use interfaces, electronic mail and bulletin boards, online connections to local, national and international databases, online connections to education administration. The use of computerbased, problem-oriented education and communication facilities between the primary health care centres and the university hospital with its laborato- 
ries and specialized departments are a great advantage.

\section{Medical data.}

As medicine is an empirical science it also relies to a great extent on medical data as carriers of medical information from individual patients tests and population studies with the important parameters as prevalence, sensitivities and specificities. Medical data is the subject matter of medical computing and as such the main target also for medical informatics. Since medical data issues are so important this is given main emphasis as an introduction but also included in many of the other parts of our courses.

Medical data are generally considered to be "soft" especially when compared to data from many other applied sciences [5]. It is therefore important to assure and ascertain adequate data quality. This is a goal to strive for, but one that probably only will be achieved gradually with better routines, procedures, questionnaires etc. A higher acceptance of using the computer for useful storage of data and a higher recognition of the computer as a very useful tool are also of importance for this goal. The users who have experienced this usefulness themselves will no doubt also be more cautious and careful in submitting only good quality data to the computer. Also more automated and supported data acquisition procedures will work in the same way.

\section{Forms of education.}

In line with the new ideas and concepts for medical education within the health university only a few lectures are given in medical informatics and those in connection with medical subjects where computers are particularly useful. The students may also choose projects as in-depth works. The aim with the in-depth work is to give the student an insight in the condition and the prerequisite of medical research and development and thereby to give them the ability to plan their own studies independently, to seek knowledge and to acquire critical appraisal skills. They will learn how to process and collect data and compare their own results with those of others and how to set up a theory and finally be able to present such a scientific work orally.

The experiences of these in-depth work has shown that in many instances the students tend to work much harder with this project, that they have been over ambitious and have made their study too comprehensive.

Below are some examples of project proposals in medical informatics:

- Evaluation of two medical expert systems, QMR, deDombal's program for acute abdominal pain, and construction of learning material based on these tools.

- Analysis of a number of medical decision situations with application of computer-based tools for decision analysis and sensitivity analysis.

- Development of computer based hypertext documents.

\section{The Medical Informatics Study Program as continuing education for health care personnel}

Based on experiences from teaching biomedical engineering and medical informatics to technical and medical students, a study program in medical informatics has been developed for health care personnel. The study program, which has been developed in co-operation with neighboring hospitals, corresponds to 20 weeks of fulltime study on a University level, and is composed of six sub courses. The basis for course development have been the process oriented view of medical informatics used in our medical informatics courses (see figure 2), and national and international reports on experiences derived from medice informatics curricula [6] at differen levels.

The Medical Informatics Study Pro gram is composed of six courses with the following content (see figure 3):

\section{1) Introduction to computer system} ( 3 credits) ( 1 credit corresponds to one full study-week)

The aim of the first course is to give basic understanding about the com. puter as a device and a tool. A com. puter (an old one, no longer used) is taken apart and its components, including peripheral devices, are ex. amined and discussed from an enduser point of view. The process of executing a program is briefly ex. plained, including the operating system and different programmin languages. Common software such as word processors, spreadsheets, graphical programs and communication tools are then presented. A significant part of the course is dedicated to hands-on work with spread. sheets and databases, and methods for.program and object integration such as Object Linking \& Embedding (OLE) and Dynamic Data Ex. change (DDE). A short introduc tion to program and system development then concludes the first course.

2) Medical data and medical termil nology ( 3 credits)

This course deals with the variout types of medical data, their origin acquisition, storage and use, and sources of inaccuracy and variability. Technical data types such as numbers, text, signals, images, sound, and other complex data types are presented. The concept of digitalization is presented along with ideas of sampling frequency, resolution, precision and accuracy. Fol lowing this presentation of technir cal data types, medical data associr 
ated with medical terminology and the structure of international normenclatures and coding schemes such as MeSH, SNOMED, EUCLIDES are then presented. The course contains a project with the objective of documenting the terminology used within a small medical domain and implementing a medical entity dictionary within a database system. The term data model is to capture synonyms, lexical variants, local history notes, etc., including references to at least one international classification.

\section{3)Information processing formedical} decision support ( 3 credits)

The aim of this course is to provide sufficient knowledge about descriptive and comparative statistics to make it possible to perform basic data analysis using databases, spreadsheets and statistical packages. Concepts from medical decision making theory such as probabilities, prevalence and predictive power are presented, and Bayes' theory and decision tree analysis are practiced. The course contains problem solving and data analysis with statistical programs and spreadsheets and exploration of knowledge based systems such as QMR and ILIAD.

\section{4) Medical information systems (5 credits)}

This course gives an overview of different medical information systems (laboratory information systems, patient monitoring systems, radiology information systems, etc.) with a focus on the computer-based patient record. The structure of the patient record and the requirements for data dictionaries (medical entity dictionaries) are discussed in particular. The client-server architecture is presented, and communication standards such as EDIFACT for medical data transfer between different health care providers is introduced. Methods and tools for information retrieval and communication between individuals and departments are also covered. The course contains a research project for which the student selects a relevant topic and does a review of recent progress based on proceedings from major international conferences such as MEDINFO and SCAMC.

\section{5) Health care organization ( 2 cred-} its)

This course gives a perspective of challenges and changes within health care organization, in which the concept of human service organizations, organization analysis, and quality assurance are presented and discussed. The non-technical per- spective is used as a basis for discussion of how to best utilize modern information technology within the health care sector.

\section{6) Project ( 3 credits)}

The concluding part of the study program is a project which is relevant to the individual's own professional workingenvironment. The type of project is thus not specified by the tutor, but rather is discussed and defined in conjunction with the student, the tutor, and the head of the department where the project is to be carried out. The project is then presented orally and in a written report. The oral presentation is done with an opponent from the same course providing a critical evaluation of the project.

\section{Medical Informatics Study Programme}

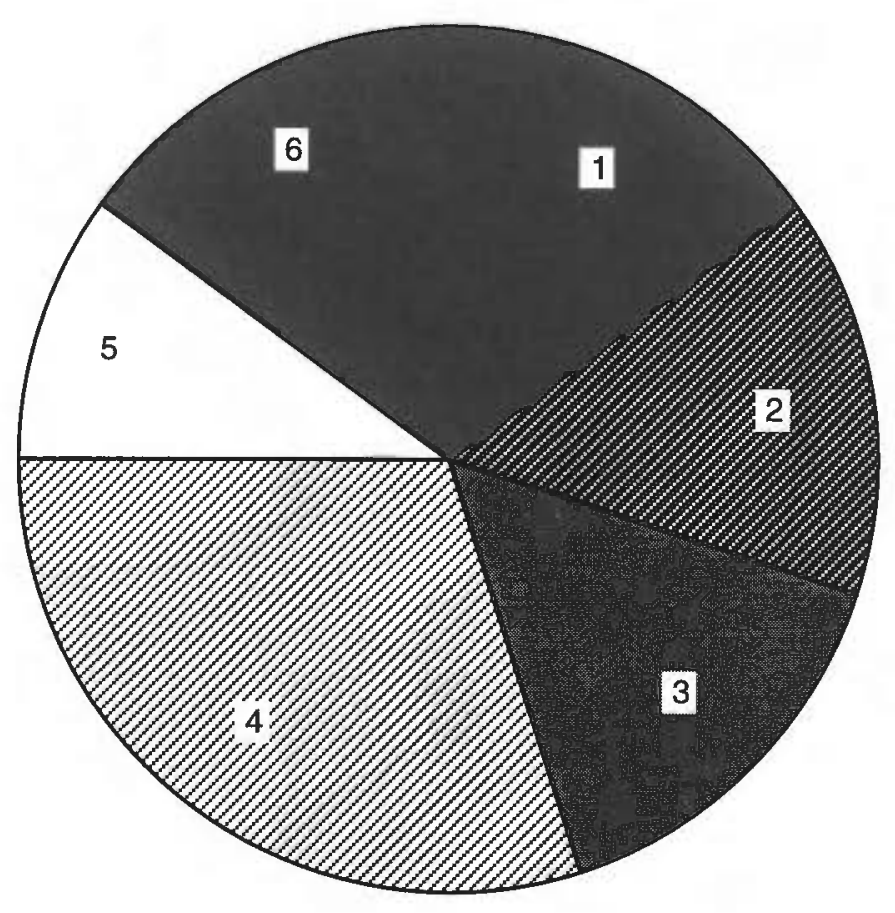

Figure 3. Composition of the study program in medical informatics ( 20 credits) for health care professionals, primarily nurses and clinical medical secretaries.

1) Introduction to computer systems, 2) Medical data and medical terminology, 3) Information processing for medical decision support, 4) Medical information systems, 5) Health care organization, 6) Project. 


\section{Discussion}

Education in biomedical engineering and medical informatics should always relate to available and used technology and methods within health care. Up to now it seems as if the interest and incentive have been more on engineering and computer sciences. The medical teachers have always had to struggle with finding space for an ever growing medical knowledge base. The adoption of problem based learning in medical schools has however evoked an awareness of formal methods as a tool to attain critical appraisal skills. This has had the effect that also our technical courses have been better recognized and asked for within the health university.

A major concern when administering continuing education for health care professionals is the allocation of resources for the studies. Without a real commitment to the study program on the part of both the individual and the organization (e.g. hospital, clinic or department) where the student is employed, there will always be insufficient time dedicated to the program. It is clearly advantageous if practical hands-on training takes place within the hospital area, and if the same hardware and software used in the courses are also available in the various departments so that individual training can be continued in direct contact with the working environment. The hardware used has primarily consisted of PCs and Macintosh computers equipped with standard desk-top software or else used as clients or termi- nals for different hospital information systems. The core of teachers in the study program comes from the Department of Medical Informatics, although teachers from other departments within the university and the university hospital are also involved in specific parts of the program. In addition to course material from the department (mostly in Swedish) Medical Informatics by Shortliffe and Perrault [7] is also used. This book provides a historical perspective concerning the use of computers in medicine and an overview of computer applications in different domains of health care, although some material is not totally relevant due to rapid technological developments a nd national differences in health care organization. An interesting issue involved in designing a continuing education program concerns the length of the program and the relative importance of theory, methods and practical handson training. It has not been our intention to cover all existing information systems in use in different hospitals and primary health care centres, but rather to teach general concepts and theories and to develop a critical perspective concerning both traditionaland IT-based information management within health care. The length of the study program is also an issue for discussion: we have given shorter introductory courses of 5 weeks, but the longer program naturally provides a much more solid platform for active participation in future organizational development, and for assuming responsibility for the installation pro- cess of different computer systeme and applications. It also provides stu. dents with a good basis to become teachers in local education programs in the departments, units or clinice concerned.

\section{References}

1. Wigertz O, Persson J, Åhlfeldt H. Teaching medical informatics to biomedical engineering students: experiences over 15 years. Meth Inform Med 1989; 4:309-12.

2. Wigertz O, Gill H, Åhlfeldt H. Teachin medical informatics to medical students. If Proc. Int. Conference on Medical Informatics and Medical Education, Praguo 1990, MIA, Elsevier Science Publish, 41. 50.

3. Åhlfeldt $\mathrm{H}$, et al. Information retrieval and computer communication within the fac. ulty of health sciences in Linköping, Swe. den. 6th Symp. on Human Interface 1990, Tokyo, 499-506.

4. Wigertz O, Elfstrom J, Hagglund S, Rosin O. Computer-assisted training in patient management and clinical decision making. Methodological approach and experiences, In: Pages J-C, Levy AH, Gremy F, Anderson J, eds. Meeting the Challenge: Informatics \& Medical Education, Elsevier, 1983.

5. Komaroff AL. The variability and inacer. racy of medical data. In: Proc. IEEE (1979), 67:1196-1207.

6. Van Bemmel JH, Zvarova J. Knowledge, Information and Medical Education. North Holland 1991.

7. Shortliffe T, Perrault. Medical Informatics: Computer Applications in Health Care. Addison Wesley, 1990.

Address of the authors:

Hans Åhlfeldt and Ove Wigertz,

Dept of Medical Informatics,

Linköping University,

S-581 85 Linköping,

Sweden 